\title{
ON EXISTENCE OF SOLUTIONS TO STOCHASTIC DIFFERENTIAL EQUATIONS WITH CURRENT VELOCITIES
}

S.V. Azaria, Kuban State University, Krasnodar, Russian Federation, azarinas@mail.ru, Yu.E. Gliklikh, Voronezh State University, Voronezh, Russian Federation, yeg@math.vsu.ru

The notion of mean derivatives was introduced by E. Nelson in 60-th years of XX century and at the moment there are a lot of mathematical models of physical processes constructed in terms of those derivatives. The paper is devoted to investigation of stochastic differential equations with current velocities, i.e., with Nelson's symmetric mean derivatives. Since the current velocities of stochastic processes are natural analogues of ordinary physical velocities of deterministic processes, such a research is important for investigation of models of physical processes that take into account stochastic properties. An existence of solution theorem for those equations is obtained.

Keywords: mean derivatives; equations with current velocities; existence and uniqueness of solutions

Introduction. The notion of mean derivatives was introduced by E. Nelson [1-3] for the needs of the so-called Nelson's stochastic mechanics (a version of quantum mechanics). Later a lot of applications of mean derivatives to some other branches of science were found. It should be pointed out that among Nelson's mean derivatives (forward, backward, symmetric and antisymmetric, etc.) the symmetric derivatives called current velocities, play the role of natural analogue of physical velocity of deterministic processes. That is why inestigation of equations with with current velocities is very important for stochastic models for many physical processes.

In this paper we investigate those equations and obtain an existence and uniqueness theorem for their solutions.

Some remarks on notations. In this paper we deal with equations and inclusions in the linear space $\mathbb{R}^{n}$, for which we always use coordinate presentation of vectors and linear operators. Vectors in $\mathbb{R}^{n}$ are considered as columns. If $X$ is such a vector, the transposed row vector is denoted by $X^{*}$. Linear operators from $\mathbb{R}^{n}$ to $\mathbb{R}^{n}$ are represented as $n \times n$ matrices, the symbol $*$ means transposition of a matrix (pass to the matrix of conjugate operator). The space of $n \times n$ matrices is denoted by $L\left(\mathbb{R}^{n}, \mathbb{R}^{n}\right)$.

By $\mathrm{S}(n)$ we denote the linear space of symmetric $n \times n$ matrices that is a subspace in $L\left(\mathbb{R}^{n}, \mathbb{R}^{n}\right)$. The symbol $\mathrm{S}_{+}(n)$ denotes the set of positive definite symmetric $n \times n$ matrices that is a convex open set in $\mathrm{S}(n)$. Its closure, i.e., the set of positive semi-definite symmetric $n \times n$ matrices, is denoted by $\overline{\mathrm{S}}_{+}(n)$.

Everywhere below for a set $B$ in $\mathbb{R}^{n}$ or in $L\left(\mathbb{R}^{n}, \mathbb{R}^{n}\right)$ the notation $\|B\|$ means sup $\|y\|$.

For the sake of simplicity we consider equations, their solutions and other objects on a finite time interval $t \in[0, T]$.

We use Einstein's summation convention with respect to shared upper and lower indices. 
1. Preliminaries on the Mean Derivatives. Consider a stochastic process $\xi(t)$ in $\mathbb{R}^{n}$, $t \in[0, l]$, given on a certain probability space $(\Omega, \mathcal{F}, \mathrm{P})$ and such that $\xi(t)$ is $L_{1}$-random variable for all $t$.

Every stochastic process $\xi(t)$ in $\mathbb{R}^{n}, t \in[0, l]$, determines three families of $\sigma$-subalgebras of $\sigma$-algebra $\mathcal{F}$ :

(i) the "past" $\mathcal{P}_{t}^{\xi}$ generated by pre-images of Borel sets in $\mathbb{R}^{n}$ by all mappings $\xi(s)$ : $\Omega \rightarrow \mathbb{R}^{n}$ for $0 \leq s \leq t$

(ii) the "future" $\mathcal{F}_{t}^{\xi}$ generated by pre-images of Borel sets in $\mathbb{R}^{n}$ by all mappings $\xi(s): \Omega \rightarrow \mathbb{R}^{n}$ for $t \leq s \leq l$;

(iii) the "present" ("now") $\mathcal{N}_{t}^{\xi}$ generated by pre-images of Borel sets in $\mathbb{R}^{n}$ by the mapping $\xi(t)$.

All families are supposed to be complete, i.e., containing all sets of probability 0 .

For convenience we denote the conditional expectation of $\xi(t)$ with respect to $\mathcal{N}_{t}^{\xi}$ by $E_{t}^{\xi}(\cdot)$.

Ordinary ("unconditional") expectation is denoted by $E$.

Strictly speaking, almost surely (a.s.) the sample paths of $\xi(t)$ are not differentiable for almost all $t$. Thus its "classical" derivatives exist only in the sense of generalized functions. To avoid using the generalized functions, following Nelson (see, e.g., [1-3]) we give

Definition 1. (i) Forward mean derivative $D \xi(t)$ of $\xi(t)$ at time $t$ is an $L_{1}$-random variable of the form

$$
D \xi(t)=\lim _{\Delta t \rightarrow+0} E_{t}^{\xi}\left(\frac{\xi(t+\Delta t)-\xi(t)}{\Delta t}\right)
$$

where the limit is supposed to exists in $L_{1}(\Omega, \mathcal{F}, \mathrm{P})$ and $\Delta t \rightarrow+0$ means that $\Delta t$ tends to 0 and $\Delta t>0$.

(ii) Backward mean derivative $D_{*} \xi(t)$ of $\xi(t)$ at $t$ is an $L_{1}$-random variable

$$
D_{*} \xi(t)=\lim _{\Delta t \rightarrow+0} E_{t}^{\xi}\left(\frac{\xi(t)-\xi(t-\Delta t)}{\Delta t}\right)
$$

where the conditions and the notation are the same as in (i).

Note that mainly $D \xi(t) \neq D_{*} \xi(t)$, but if, say, $\xi(t)$ a.s. has smooth sample paths, these derivatives evidently coinside.

From the properties of conditional expectation (see [5] ) it follows that $D \xi(t)$ and $D_{*} \xi(t)$ can be represented as compositions of $\xi(t)$ and Borel measurable vector fields (regressions)

$$
\begin{aligned}
& Y^{0}(t, x)=\lim _{\Delta t \rightarrow+0} E\left(\frac{\xi(t+\Delta t)-\xi(t)}{\Delta t} \mid \xi(t)=x\right) \\
& Y_{*}^{0}(t, x)=\lim _{\Delta t \rightarrow+0} E\left(\frac{\xi(t)-\xi(t-\Delta t)}{\Delta t} \mid \xi(t)=x\right)
\end{aligned}
$$

on $\mathbb{R}^{n}$. This means that $D \xi(t)=Y^{0}(t, \xi(t))$ and $D_{*} \xi(t)=Y_{*}^{0}(t, \xi(t))$.

Definition 2. The derivative $D_{S}=\frac{1}{2}\left(D+D_{*}\right)$ is called symmetric mean derivative. The derivative $D_{A}=\frac{1}{2}\left(D-D_{*}\right)$ is called anti-symmetric mean derivative .

Consider the vector fields $v^{\xi}(t, x)=\frac{1}{2}\left(Y^{0}(t, x)+Y_{*}^{0}(t, x)\right)$ and $u^{\xi}(t, x)=\frac{1}{2}\left(Y^{0}(t, x)-\right.$ $\left.Y_{*}^{0}(t, x)\right)$. 
Definition 3. $v^{\xi}(t)=v^{\xi}(t, \xi(t))=D_{S} \xi(t)$ is called current velocity of $\xi(t)$; $u^{\xi}(t)=u^{\xi}(t, \xi(t))=D_{A} \xi(t)$ is called osmotic velocity of $\xi(t)$.

For stochastic processes the current velocity is a direct analogue of ordinary physical velocity of deterministic processes (see, e.g., [1-3,8]). The osmotic velocity measures how fast the "randomness" grows up.

Recall that Ito process is a process $\xi(t)$ of the form

$$
\xi(t)=\xi_{0}+\int_{0}^{t} a(s) d s+\int_{0}^{t} A(s) d w(s)
$$

where $a(t)$ is a process in $\mathbb{R}^{n}$ whose sample paths a.s. have bounded variation; $A(t)$ is a process in $L\left(\mathbb{R}^{n}, \mathbb{R}^{n}\right)$ such that for any element $A_{i}^{j}(t)$ of matrix $A(t)$ the condition $\mathrm{P}\left(\omega \mid \int_{0}^{T}\left(A_{i}^{j}\right)^{2} d t<\infty\right)=1$ holds; $w(t)$ is a Wiener process in $\mathbb{R}^{n}$; the first integral is the Lebesgue integral, the second one is Itô integral and all integrals are well-posed.

Definition 4. An Itô process $\xi(t)$ is called a process of diffusion type if a $(t)$ and $A(t)$ are not anticipating with respect to $\mathcal{P}_{t}^{\xi}$ and the Wiener process $w(t)$ is adapted to $\mathcal{P}_{t}^{\xi}$. If $a(t)=a(t, \xi(t))$ and $A(t)=A(t, \xi(t))$, where $a(t, x)$ and $A(t, x)$ are Borel measurable mappings from $[0, T] \times \mathbb{R}^{n}$ to $\mathbb{R}^{n}$ and to $L\left(\mathbb{R}^{n}, \mathbb{R}^{n}\right)$, respectively, the Itô process is called a diffusion process.

In the latter case with Borel measurable $a(t, x)$ and $A(t, x)$ process $\xi(t)$ is supposed to be a weak solution of the above equation.

Below we are dealing with smooth fields of non-degenerate linear operators $A(x)$ : $\mathbb{R}^{n} \rightarrow \mathbb{R}^{n}, x \in \mathbb{R}^{n}$ (i.e., $(1,1)$-tensor field on $\mathbb{R}^{n}$ ). Let $\xi(t)$ be a diffusion process in which the integrand under Itô integral is of the form $A(\xi(t))$. Then its diffusion coefficient $A(x) A^{*}(x)$ is a smooth field of symmetric positive definite matrices $\alpha(x)=\left(\alpha^{i j}(x)\right)((2,0)$ tensor field on $\left.\mathbb{R}^{n}\right)$. Since all these matrices are non-degenerate and smooth, there exist the smooth field of converse symmetric and positive definite matrices $\left(\alpha_{i j}\right)$. Hence this field can be used as a new Riemannian $\alpha(\cdot, \cdot)=\alpha_{i j} d x^{i} \otimes d x^{j}$ on $\mathbb{R}^{n}$. The volume form of this metric is $\Lambda_{\alpha}=\sqrt{\operatorname{det}\left(\alpha_{i j}\right)} d x^{1} \wedge d x^{2} \wedge \cdots \wedge d x^{n}$.

Denote by $\rho^{\xi}(t, x)$ the probability density of random element $\xi(t)$ with respect to the volume form $d t \wedge \Lambda_{\alpha}=\sqrt{\operatorname{det}\left(\alpha_{i j}\right)} d t \wedge d x^{1} \wedge d x^{2} \wedge \cdots \wedge d x^{n}$ on $[0, T] \times \mathbb{R}^{n}$, i.e., for every continuous bounded function $f:[0, T] \times \mathbb{R}^{n} \rightarrow \mathbb{R}$ the relation holds.

$$
\int_{0}^{T} E(f(t, \xi(t))) d t=\int_{0}^{T}\left(\int_{\Omega} f(t, \xi(t)) d \mathrm{P}\right) d t=\int_{0}^{T}\left(\int_{\mathbb{R}^{n}} f(t, x) \rho^{\xi}(t, x) \Lambda_{\alpha}\right) d t
$$

Lemma 1. $[9,10]$ Let $\xi(t)$ satisfy the Ito equation

$$
\xi(t)=\xi_{0}+\int_{0}^{t} a(s, \xi(s)) d s+\int_{0}^{t} A(s, \xi(s)) d w(s)
$$

Then

$$
u^{\xi}(t, x)=\frac{1}{2} \frac{\frac{\partial}{\partial x^{j}}\left(\alpha^{i j} \rho^{\xi}(t, x)\right)}{\rho^{\xi}(t, x)} \frac{\partial}{\partial x^{i}}
$$


where $\left(\alpha^{i j}\right)$ is the matrix of operator $A A^{*}$ under the assumption that $\rho^{\xi}(t, x)$ is smooth and nowhere equal to zero.

Remark 1. Denote by $\Xi(x)$ the vector field whose coordinate presentation is $\frac{\partial \alpha^{i j}}{\partial x^{j}} \frac{\partial}{\partial x^{i}}$. One can easily derive from (4) that $u^{\xi}(t, x)=\frac{1}{2} \operatorname{Grad} \log \rho^{\xi}(t, x)+\frac{1}{2} \Xi(x)$ where Grad denotes the gradient with respect to metric $\alpha(\cdot, \cdot)$. Indeed, $\frac{\frac{\partial}{\partial x^{j}}\left(\alpha^{i j} \rho^{\xi}(t, x)\right)}{\rho^{\xi}(t, x)} \frac{\partial}{\partial x^{i}}=\frac{1}{2} \alpha^{i j} \frac{\partial \rho^{\xi}}{\rho^{\xi}} \frac{\partial}{\partial x^{i}}+\frac{1}{2} \frac{\partial \alpha^{i j}}{\partial x^{j}} \frac{\partial}{\partial x^{i}}$ where $\alpha^{i j} \frac{\frac{\partial \partial^{\xi}}{\partial x^{j}}}{\rho^{\xi}} \frac{\partial}{\partial x^{i}}=$ Grad $\log \rho^{\xi}$ and $\frac{\partial \alpha^{i j}}{\partial x^{j}} \frac{\partial}{\partial x^{i}}=\Xi$.

Lemma 2. [3,8] For $v^{\xi}(t, x)$ and $\rho^{\xi}(t, x)$ the following interrelation

$$
\frac{\partial \rho^{\xi}(t, x)}{\partial t}=-\operatorname{Div}\left(v^{\xi}(t, x) \rho^{\xi}(t, x)\right)
$$

(known as the equation of continuity) takes place where Div denotes the divergence with respect to Riemannian metric $\alpha(\cdot, \cdot)$.

Following $[7,8]$ we introduce the differential operator $D_{2}$ that differentiates an $L_{1}$ random process $\xi(t), t \in[0, T]$ according to the rule

$$
D_{2} \xi(t)=\lim _{\triangle t \rightarrow+0} E_{t}^{\xi}\left(\frac{(\xi(t+\Delta t)-\xi(t))(\xi(t+\triangle t)-\xi(t))^{*}}{\triangle t}\right),
$$

where $(\xi(t+\triangle t)-\xi(t))$ is considered as a column vector (vector in $\left.\mathbb{R}^{n}\right),(\xi(t+\triangle t)-\xi(t))^{*}$ is a row vector (transposed, or conjugate vector) and the limit is supposed to exists in $L_{1}(\Omega, \mathcal{F}, \mathrm{P})$. We emphasize that the matrix product of a column on the left and a row on the right is a matrix so that $D_{2} \xi(t)$ is a symmetric positive semi-definite matrix function on $[0, T] \times \mathbb{R}^{n}$. We call $D_{2}$ the quadratic mean derivative.

Theorem 1. [7,8] For an Itô diffusion type process $\xi(t)$ the forward mean derivative $D \xi(t)$ exists and equals $E_{t}^{\xi}(a(t))$. In particular, if $\xi(t)$ a diffusion process, $D \xi(t)=a(t, \xi(t))$.

Theorem 2. $[7,8]$ Let $\xi(t)$ be a diffusion type process. Then $D_{2} \xi(t)=E_{t}^{\xi}[\alpha(t)]$ where $\alpha(t)=A A^{*}$. In particular, if $\xi(t)$ is a diffusion process, $D_{2} \xi(t)=\alpha(t, \xi(t))$ where $\alpha=A A^{*}$ is the diffusion coefficient.

Lemma 3. $[7,8]$ Let $\alpha(t, x)$ be a jointly continuous (measurable, smooth) mapping from $[0, T] \times \mathbb{R}^{n}$ to $\mathrm{S}_{+}(n)$. Then there exists a jointly continuous (measurable, smooth, respectively) mapping $A(t, x)$ from $[0, T] \times \mathbb{R}^{n}$ to $L\left(\mathbb{R}^{n}, \mathbb{R}^{n}\right)$ such that for all $t \in R, x \in \mathbb{R}^{n}$ the equality $A(t, x) A^{*}(t, x)=\alpha(t, x)$ holds.

2. Main Results. As it is mentioned in Section 1, the meaning of current velocities is analogous to that of ordinary velocity for a non-random process. Thus the case of equations with current velocities is probably the most natural from the physical point of view.

The system of the form

$$
\left\{\begin{array}{l}
D_{S} \xi(t)=v(t, \xi(t)) \\
D_{2} \xi(t)=\alpha(t, \xi(t))
\end{array}\right.
$$

is called a first order differential equation with current velocities. 
Definition 5. We say that (7) has a solution on the interval $[0, T]$ if there exists a probability space $(\Omega, \mathcal{F}, \mathrm{P})$ and a process $\xi(t)$ given on $(\Omega, \mathcal{F}, \mathrm{P})$ for $t \in[0, T]$, that satisfies (7).

Theorem 3. Let $v:[0, T] \times \mathbb{R}^{n} \rightarrow \mathbb{R}^{n}$ be smooth and $\alpha: \mathbb{R}^{n} \rightarrow \mathrm{S}_{+}(n)$ be smooth and autonomous (so, it determines the Riemannian metric $\alpha(\cdot, \cdot)$ on $\mathbb{R}^{n}$, introduced in Section 1). Let them also satisfy the estimates

$$
\begin{gathered}
\|v(t, x)\|<K(1+\|x\|), \\
\operatorname{tr} \alpha(x)<K\left(1+\|x\|^{2}\right)
\end{gathered}
$$

and for all indices ij let the elements of matrix $\alpha(x)$ satisfy the inequality

$$
\left|\frac{\partial \alpha^{i j}}{\partial x^{j}}(x)\right|<K(1+\|x\|)
$$

for some $K>0$. Let $\xi_{0}$ be a random element with values in $\mathbb{R}^{n}$ whose probability density $\rho_{0}$ with respect to the volume form $\Lambda_{\alpha}$ of $\alpha(\cdot, \cdot)$ on $\mathbb{R}^{n}$ (see Section 1), is smooth and nowhere equal to zero. Then for the initial condition $\xi(0)=\xi_{0}$ equation (7) has a solution that is well posed on the entire interval $t \in[0, T]$ and unique as a diffusion process.

Proof. Since $v(t, x)$ is smooth and estimate (8) is fulfilled, its flow $g_{t}$ is well posed on the entire interval $t \in[0, T]$. By $g_{t}(x)$ we denote the orbit of the flow (i.e., the solution of equation $\left.x^{\prime}(t)=v(t, x)\right)$ with the initial condition $g_{0}(x)=x$. Since $v(t, x)$ is smooth, its flow is also smooth.

Continuity equation (5) obviously can be transformed into the form

$$
\frac{\partial \rho}{\partial t}=-\alpha(v, \operatorname{Grad} \rho)-\rho \operatorname{Div} v
$$

Suppose that $\rho(t, x)$ nowhere in $[0, T] \times \mathbb{R}^{n}$ equals zero. Then we can divide (11) by $\rho$ so that it is transformed into the equation

$$
\frac{\partial p}{\partial t}=-\alpha(v, \text { Grad } p)-\operatorname{Div} v
$$

where $p=\log \rho$. Introduce $p_{0}=\log \rho_{0}$. Show that the solution of (12) with initial condition $p(0)=p_{0}$ is described by the formula $p(t, x)=p_{0}\left(g_{-t}(x)\right)-\int_{0}^{t}(\operatorname{Div} v)\left(s, g_{s}\left(g_{-t}(x)\right) d s\right.$. Introduce the product $[0, T] \times \mathbb{R}^{n}$ and consider the function $p_{0}$ as given on the level surface $\left(0, \mathbb{R}^{n}\right)$. Consider the vector field $(1, v(t, x))$ on $[0, T] \times \mathbb{R}^{n}$. The orbits of its flow $\hat{g}_{t}$, starting at the points of $\left(0, \mathbb{R}^{n}\right)$, have the form $\hat{g}_{t}(0, x)=\left(t, g_{t}(x)\right)$ and the flow is smooth as well as $g_{t}$. Also introduce on $[0, T] \times \mathbb{R}^{n}$ the Riemannian metric $\hat{\alpha}(\cdot, \cdot)$ by the formula $\hat{\alpha}\left(\left(X_{1}, Y_{1}\right),\left(X_{2}, Y_{2}\right)\right)=X_{1} X_{2}+\alpha\left(Y_{1}, Y_{2}\right)$. Notice that for any $(t, x)$ the point $\hat{g}_{-t}(t, x)$ belongs to $\left(0, \mathbb{R}^{n}\right)$ where the function $p_{0}$ is given. Thus on the one hand $(1, v) p(t, x)$, the derivative of $p(t, x)$ in the direction of $(1, v)$, by construction equals $-\operatorname{Div} v(t, x)$. And on the other hand one can easily calculate that $(1, v) p(t, x)=$ $\frac{\partial}{\partial t} p(t, x)+\alpha(v(t, x)$, Grad $p(t, x))$. Thus (12) is satisfied.

Notice that $\rho=e^{p}$ is indeed nowhere zero and so our arguments are well-posed. From the construction it follows that for a given field $\alpha$ and initial density $\rho_{0}$ satisfying the hypothesis, the densities of constructed type and the smooth vector fields having compete flows, are in one-to-one correspondence. Thus after finding the density $\rho(t, x)$ 
for the solution of (7), we can find also the osmotic velocity $u^{\xi}(t, x)$ by formula (4), i.e., $u=\frac{1}{2} \operatorname{Grad} p+\frac{1}{2} \Xi=\operatorname{Grad} \log \sqrt{\rho}+\frac{1}{2} \Xi$. Note that $u$ is uniquely determined by $\rho$ and $\alpha$ and so the forward mean derivative of the solution is also uniquely determined by the formula $a(t, x)=v(t, x)+\frac{1}{2} \operatorname{Grad} p+\frac{1}{2} \Xi=\operatorname{Grad} \log \sqrt{\rho}+\frac{1}{2} \Xi$. From Lemma 3 and from the hypothesis of Theorem it follows that there exists smooth $A(x)$ such that $A(x) A^{*}(x)=\alpha(x)$ and the relation $\|A(t, x)\|<K(1+\|x\|)$ holds. Then from the general theory of equations with forward mean derivatives it follows that $\xi(t)$ having the density $\rho(t, x)$ as above must satisfy the stochastic differential equation

$$
\xi(t)=\xi_{0}+\int_{0}^{t} a(s, \xi(s)) d s+\int_{0}^{t} A(s, \xi(s)) d w(s) .
$$

From the hypothesis and from results of [4] it follows that (13) has has a unique strong solution $\xi(t)$ with initial density $\rho_{0}$ well-posed for $t \in[0, T]$. Thus, by Theorem $2 D_{2} \xi(t)=$ $\alpha(\xi(t))$. The fact that $D_{S} \xi(t)=v(t, \xi(t))$ follows from the construction.

Lemma 4. Let $\alpha(x), \rho(t, x)$ and $\Lambda_{\alpha}$ be the same as in Theorem 3. Let also the vector field $v$ from Theorem 3 be autonomous. Then the flow $\hat{g}_{t}$ of vector field $(1, v(x))$ on $[0, T] \times \mathbb{R}^{n}$ preserves the volume form $\rho(t, x) d t \wedge \Lambda_{\alpha}$ (i.e., $\hat{g}_{t}^{*}\left(\rho(t, x) d t \wedge \Lambda_{\alpha}\right)=\rho_{0}(x) d t \wedge \Lambda_{\alpha}$ where $\hat{g}_{t}^{*}$ is the pull back) and so for any measurable set $Q \subset \mathbb{R}^{n}$ and for any $t \in[0, T]$

$$
\int_{Q} \rho_{0}(x) \Lambda_{\alpha}=\int_{g_{t}(Q)} \rho(t, x) \Lambda_{\alpha} .
$$

Proof. It is enough to show that $L_{(1, v)}\left(\rho(t, x) d t \wedge \Lambda_{\alpha}\right)=0$ where $L_{(1, v)}$ is the Lie derivative along $(1, v)$. Obviously

$$
L_{(1, v)}\left(\rho(t, x) d t \wedge \Lambda_{\alpha}\right)=\left(L_{(1, v)} \rho(t, x)\right) d t \wedge \Lambda_{\alpha}+\rho(t, x)\left(L_{(1, v)} d t \wedge \Lambda_{\alpha}\right) .
$$

For a function the Lie derivative coincides with the derivative in direction of vector field, hence $L_{(1, v)} \rho(t, x)=\frac{\partial \rho}{\partial t}+\alpha(v, G r a d \rho)$ (see the proof of Theorem 3) and so $\left(L_{(1, v)} \rho(t, x)\right) d t \wedge$ $\Lambda_{\alpha}=\left(\frac{\partial \rho}{\partial t}+\alpha(v, \operatorname{Grad} \rho)\right) d t \wedge \Lambda_{\alpha}$. Since neither the form $\Lambda_{\alpha}$ nor the vector filed $v(x)$ depend on $t, L_{(1, v)} d t \wedge \Lambda_{\alpha}=d t \wedge\left(L_{v} \Lambda_{\alpha}\right)=\operatorname{Div} v\left(d t \wedge \Lambda_{\alpha}\right)$ as the Lie derivative along $v$ of the volume form $\Lambda_{\alpha}$ equals (Divv) $\Lambda_{\alpha}$ (see, e.g., [6]). Taking into account (11), we obtain $L_{(1, v)}\left(\rho(t, x) d t \wedge \Lambda_{\alpha}\right)=0$.

\section{References}

1. Nelson E. Derivation of the Schrödinger Equation from Newtonian Mechanics. Physical Review, 1966, vol. 150, no. 4, pp. 1079-1085. DOI: 10.1103/PhysRev.150.1079

2. Nelson E. Dynamical Theory of Brownian Motion. Princeton, Princeton University Press, 1967. $142 \mathrm{p}$.

3. Nelson E. Quantum Fluctuations. Princeton, Princeton University Press, 1985. 147 p.

4. Gihman I.I., Skorohod A.V. Theory of Stochastic Processes. V. 3. New York, SpringerVerlag, 1979. DOI: 10.1007/978-1-4615-8065-2 [Гихман, И.И. Теория случайных процессов / И.И. Гихман, А.В. Скороход. - М.: Наука, 1975. - Т. 3. - 496 с.]

5. Parthasarathy K.R. Introduction to Probability and Measure. New York, Springer-Verlag, 1978. [Партасарати, К. Введение в теорию вероятностей и теорию меры. - М.: Мир, 1988. - 343 c.] 
6. Schutz B.F. Geometrical Methods of Mathematical Physics. Cambridge, Cambridge University Press, 1982. [Шутц, Б. Геометрические методы математической физики / Б. Шутц. - М.: Мир, 1984. - 303 c.]

7. Azarina S.V., Gliklikh Yu.E. Differential Inclusions with Mean Derivatives. Dynamic Systems and Applications, 2007, vol. 16, no. 1, pp. 49-71.

8. Gliklikh Yu.E. Global and Stochastic Analysis with Applications to Mathematical Physics. London, Springer-Verlag, 2011. 460 p. DOI: 10.1007/978-0-85729-163-9

9. Cresson J., Darses S. Stochastic Embedding of Dynamical Systems. Journal of Mathematical Physics, 2007, vol. 48, pp. 072703-1-072303-54. DOI: 10.1063/1.2736519

10. Gliklikh Yu.E., Mashkov E.Yu. Stochastic Leontieff Type Equations and Mean Derivatives of Stochastic Processes. Bulletin of the South Ural State University. Series: Mathematical Modelling, Programming and Computer Software, 2013, vol. 6, no. 2, pp. 25-39. [Gliklikh, Yu.E. Stochastic Leontieff Type Equations and Mean Derivatives of Stochastic Processes / Yu.E. Gliklikh, E.Yu. Mashkov // Вестник ЮУрГУ. Серия: Математическое моделирование и программирование. - 2013. - Т. 6, № 2. - С. 25-39.]

This research is supported in part by Russian Scientific Foundation (RSCF) Grant 14-21-00066, being carried out by Voronezh State University.

Received May 21, 2015

УДК $517.9+519.216 .2$

DOI: $10.14529 / \mathrm{mmp} 150408$

\title{
О СУЩЕСТВОВАНИИ РЕШЕНИЙ СТОХАСТИЧЕСКИХ ДИФФЕРЕНЦИАЛЬНЫХ УРАВНЕНИЙ С ТЕКУЩИМИ СКОРОСТЯМИ
}

\author{
С.В. Азарина, Ю.Е. Гликлих
}

Понятия производных в среднем были введены Э. Нельсоном в 60-х годах ХХ века, и в настоящий момент имеется много математических моделей физических процессов, построенных в терминах этих производных. Статья посвящена исследованию стохастических дифференциальных уравнений с текущими скоростями, т.е., с нельсоновскими симметрическими производными в среднем. Поскольку текущие скорости случайных процессов являются естественными аналогами обычных физических скоростей детерминированных процессов, изучение таких уравнений важно для исследования моделей физических процессов, которые учитывают стохастические свойства. Получена теорема существования решения для указанного типа уравнений.

Ключевые слова: производные в среднем; уравнения с текущими скоростями; существование и единственность решений.

Светлана Владимировна Азарина, кандидат физико-математических наук, доцент, Кубанский государственный университет (г. Краснодар, Российская Федерация), azarinas@mail.ru.

Юрий Евгеньевич Гликлих, доктор физико-математических наук, профессор, кафедра «Алгебра и топологические методы анализа», Воронежский государственный университет (г. Воронеж, Российская Федерация), уeg@math.vsu.ru.

Поступила в редакцию 21 мая 2015 г. 